\title{
Developments in the meiotic analysis of hybrids. III. Amended models for pentaploids
}

\author{
C. G. D. CHAPMAN \& G. KIMBER* \\ $P B 1$ Cambridge, Masis Lane, Trumpington, Cambridge CB2 2LO, UK and *Department of Agronomy, Curtis Hall, \\ University of Missouri-Columbia, MO 65211, USA
}

\begin{abstract}
Amended models of meiotic behaviour in pentaploid hybrids are presented, which better represent the theory and assumptions about chromosome pairing that such models necessarily incorporate. They correct problems concerning the distribution of chiasmata among and within chromosome configurations inherent in earlier models. In general these analyses give similar interpretations to earlier models but in some cases suggest differing conclusions. The extent of pairing possibilities at the pentaploid level can make it difficult to choose with confidence among the proposed models when the level of chromosome pairing is low.
\end{abstract}

Keywords: genome analysis, meiotic models, pentaploids.

\section{Introduction}

The analysis of meiosis in interspecific hybrids is an effective way to assess genome relationships amongst species. It compares a comparatively large amount of DNA, well dispersed within and among chromosomes (Kimber \& Yen, 1990), whilst being technically undemanding in terms of equipment and supplies. The necessary conditions for its application are that the hybrid to be investigated is polyploid to allow the opportunity for preferential pairing, that the cross can be made and the plants brought to flowering, and that the chromosome figures formed at metaphase I of meiosis can be identified. In this series of papers the models for meiotic analysis developed by Alonso \& Kimber (1981), Kimber \& Alonso (1981) and Espinasse \& Kimber (1981) for triploid, tetraploid and pentaploid hybrids, respectively, are being re-examined and extended.

Chapman \& Kimber (1992a) demonstrated an advantage in weighting meiotic figure frequencies by the number of chromosomes each contains when calculating the merit function to be minimized during optimization. The use of this weighted sums of squares of difference (WSSD), rather than the simple sums of squares of differences (SSD), between observed and calculated figure frequencies removes a bias in the estimation of $x$, the measure of relative affinity, towards values favoured by the frequency of the smaller meiotic figures.

Chapman \& Kimber (1992b) also dealt with two problems in the earlier tetraploid models of Kimber \&
Alonso (1981). These concerned the placement of the second pair of bound arms within a configuration of four homoeologous chromosomes, and the distribution of arm pairing amongst homoeologous groups. The practical result of these problems was the failure of these models to match expected meiotic patterns in two cases (the 2:1:1 and $3: 1$ models) and to give ambiguous results in the third $(2: 2$ model). These deficiencies could lead to erroneous interpretations of real datasets. New models were developed to correct these problems which, in some cases, lead to differing conclusions about the pattern of pairing in species hybrids.

Espinasse \& Kimber (1981), developed formulae to describe five basic situations for the analysis of pentaploid hybrids. The first of these, designated the $2: 2: 1$ model, has two pairs of genomes considered to be equally and more closely related, with the remaining relationships also being equal but more distant (Fig. 1). Similarly the $3: 2$ model has a triplet and a pair of more closely related genomes; the $2: 1: 1: 1$ a single pair of more closely related genomes; the $3: 1: 1$ a single triplet of more closely related genomes; and the $4: 1$ a quartet of more closely related genomes. The remaining relationships are considered more distant but equal (Fig. 1). A sixth case, the 5:0 model, corresponds to the situation where all five genomes are equally related. At extremes it may be illustrated by the genomic formulae AAAAA where the genomes are identical, or $\mathrm{ABCDE}$ where they are equally unrelated. It represents a special case of the other five models when they converge on equal affinity amongst all the genomes present. 


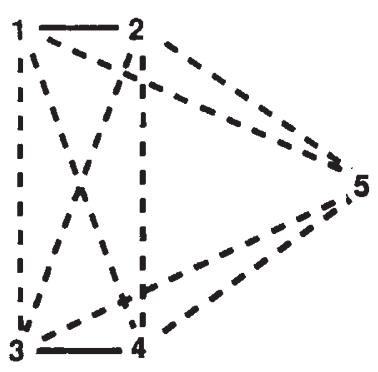

2:2:1

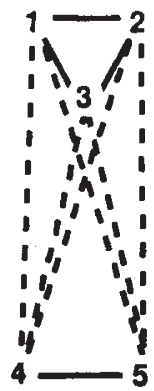

$3: 2$

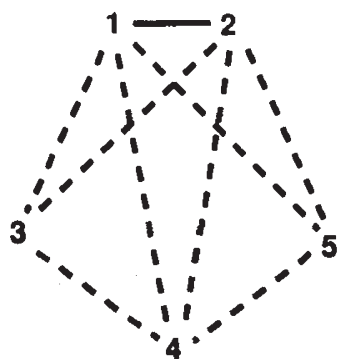

2:1:1:1

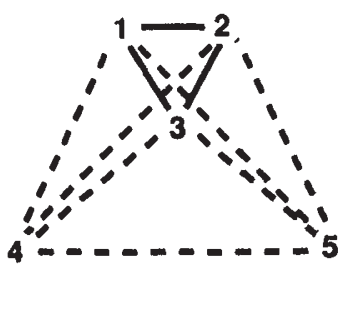

$3: 1: 1$

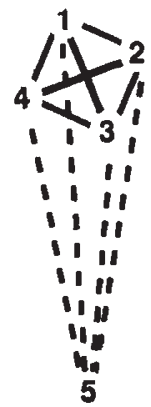

$4: 1$

Fig. 1 The five basic models for genome relationships in pentaploid hybrids. (_- More closely related genomes, relative affinity $=x,(---)$ less closely related genomes, relativeaffinity $=y$.

However, the two basic problems of placing arm pairing within and among homoeologous groups that affected the tetraploid models also perturb the pentaploid ones. Table 1 illustrates this by proposing ideal datasets for the various models and then analysing them by the models of Espinasse \& Kimber (1981). The ideal dataset for the 2:2:1 model, for example, where pairing is confined to and complete within two pairs of genomes, is a ratio of two ring bivalents to one univalent. The application of those models to ideal datasets for the six different genomic situations outlined above leads to a poor fit for the $2: 1: 1: 1$ and $3: 1: 1$ models and an ambiguous solution for the $3: 2$ model (Table 1). Their application to real datasets must therefore also be suspect.

These weaknesses are addressed below through the reconstruction of the pentaploid pairing models, and the incorporation of the amended approach to optimization. The terms pairing, meiotic figure, meiotic configuration, arm configuration, dataset, meiotic analysis

Table 1 Comparison of the present models with those of Espinasse \& Kimber (1981) for idealized datasets for pentaploid hybrids with a basic number of 7

\begin{tabular}{|c|c|c|c|c|c|c|c|c|c|c|c|c|}
\hline Genomes & Model & $I^{*}$ & \lfloor & (II) & III & $\lfloor$ IV & (IV) & V & $c$ & \multicolumn{2}{|c|}{ Merit function } & $x$ \\
\hline \multirow{3}{*}{$\begin{array}{l}\text { 1. AAAAA or } \\
\text { ABCDE }\end{array}$} & Ideal 5:0 & 1.40 & 0.00 & 2.80 & 1.87 & 0.00 & 0.93 & 3.73 & 1.000 & & & \\
\hline & $\mathrm{E} / \mathrm{K}+5: 0$ & 1.40 & 0.00 & 2.80 & 1.87 & 0.00 & 0.93 & 3.73 & & SSD $\ddagger$ & 0.000 & 0.500 \\
\hline & $\mathrm{C} / \mathrm{K} 5: 0$ & 1.40 & 0.00 & 2.80 & 1.87 & 0.00 & 0.93 & 3.73 & & WSSD & 0.000 & 0.500 \\
\hline \multirow[t]{3}{*}{ 2. $\mathrm{AABBC}$} & Ideal $2: 2: 1$ & 7.00 & 0.00 & 14.00 & 0.00 & 0.00 & 0.00 & 0.00 & 1.000 & & & \\
\hline & $\mathrm{E} / \mathrm{K} 2: 2: 1$ & 7.00 & 0.00 & 14.00 & 0.00 & 0.00 & 0.00 & 0.00 & & SSD & 0.000 & 1.000 \\
\hline & $\mathrm{C} / \mathrm{K} 2: 2$ & 7.00 & 0.00 & 14.00 & 0.00 & 0.00 & 0.00 & 0.00 & & WSSD & 0.000 & 1.000 \\
\hline \multirow[t]{5}{*}{ 3. $\mathrm{AAABB}$} & Ideal 3:2 & 2.33 & 0.00 & 9.33 & 4.67 & 0.00 & 0.00 & 0.00 & 1.000 & & & \\
\hline & $\mathrm{E} / \mathrm{K} 3: 2$ & 2.33 & 0.00 & 9.33 & 4.67 & 0.00 & 0.00 & 0.00 & & SSD & 0.000 & 1.000 \\
\hline & $\mathrm{E} / \mathrm{K} 2: 1: 1: 1$ & 2.33 & 0.00 & 9.33 & 4.67 & 0.00 & 0.00 & 0.00 & & SSD & 0.000 & 1.000 \\
\hline & $\mathrm{C} / \mathrm{K} 3: 2$ & 2.33 & 0.00 & 9.33 & 4.67 & 0.00 & 0.00 & 0.00 & & WSSD & 0.000 & 1.000 \\
\hline & C/K 2:1:1:1 & 5.35 & -3.59 & 5.76 & 1.34 & 1.51 & 0.82 & 2.39 & & WSSD & 401.346 & 0.861 \\
\hline \multirow[t]{3}{*}{ 4. $\mathrm{AABCD}$} & Ideal $2: 1: 1: 1$ & 21.00 & 0.00 & 7.00 & 0.00 & 0.00 & 0.00 & 0.00 & 0.500 & & & \\
\hline & $\mathrm{E} / \mathrm{K} 2: 1: 1: 1$ & 17.16 & 3.53 & 4.92 & 0.31 & 0.01 & 0.00 & 0.00 & & SSD & 31.636 & 1.000 \\
\hline & $\mathrm{C} / \mathrm{K} 2: 1: 1: 1$ & 21.00 & 0.00 & 7.00 & 0.00 & 0.00 & 0.00 & 0.00 & & WSSD & 0.000 & 1.000 \\
\hline \multirow[t]{3}{*}{ 5. $\mathrm{AAABC}$} & Ideal $3: 1: 1$ & 16.33 & 0.00 & 2.33 & 4.67 & 0.00 & 0.00 & 0.00 & 0.500 & & & \\
\hline & $\mathrm{E} / \mathrm{K} 3: 1: 1$ & 15.03 & 2.73 & 1.68 & 2.27 & 0.78 & 0.06 & 0.19 & & SSD & 16.003 & 1.000 \\
\hline & C/K $3: 1: 1$ & 16.33 & 0.00 & 2.33 & 4.67 & 0.00 & 0.00 & 0.00 & & WSSD & 0.000 & 1.000 \\
\hline \multirow[t]{3}{*}{ 6. $\mathrm{AAAAB}$} & Ideal 4:1 & 7.00 & 0.00 & 4.67 & 0.00 & 0.00 & 4.67 & 0.00 & 1.000 & & & \\
\hline & E/K 4:1 & 7.00 & 0.00 & 4.67 & 0.00 & 0.00 & 4.67 & 0.00 & & SSD & 0.000 & 1.000 \\
\hline & $\mathrm{C} / \mathrm{K} 4: 1$ & 7.00 & 0.00 & 4.67 & 0.00 & 0.00 & 4.67 & 0.00 & & WSSD & 0.000 & 1.000 \\
\hline
\end{tabular}

*I = univalents $\lfloor$ II $\rfloor$ = rod bivalents; II $=$ ring bivalents; III = trivalents; $\lfloor$ IV $\rfloor=$ chain quadrivalents; (IV) = closed quadrivalents; $\mathrm{V}=$ quinquevalents. $\dagger \mathrm{E} / \mathrm{K}=$ analysis of the ideal data by the models of Espinasse \& Kimber $(1981) ; \mathrm{C} / \mathrm{K}=$ analysis of the ideal data by the present models. $\Varangle S S D=$ Sums of squares of difference; WSSD = weighted sums of squares of difference. 
and $c$ are used as discussed by Chapman \& Kimber (1990a).

\section{Theory}

As with the triploid and tetraploid models, a number of assumptions are made about the nature of chromosome pairing (Chapman \& Kimber 1992a). Briefly, these are that the two arms of a chromosome are independent in their pairing behaviour; that the affinities and tendency to pair among any set of five homoeologous arms are also those among the entire genomes; that the pairing of any two arms precludes the involvement of a third; and that there is no effect of translocations in generating multivalents. Thus, the overall meiotic behaviour expected can be derived from considering how a single set of five homoelogous arms pair.

The pairing possibilities and their consequences are considerably more complex at the pentaploid level than at the tetraploid. For example, there are 10 rather than six pairwise relationships, leading ultimately to 12 rather than nine meiotic configurations. To carry out the correspondingly elaborate meiotic analysis for the five basic pentaploid models, a computer program was written in TURBo PASCAL for the Apple Macintosh. It may be described as an inelegant, 'brute force' approach, because it calculates the probability of each possible pattern of arm pairing, and hence all the possibilities (676) of pairing for one set of five homoeologous chromosomes. The calculations relating to one set of homoeologous arms are detailed in the Appendix, while below are outlined the principles on which the pentaploid models have been constructed.

The affinity relationships among the five genomes in a pentaploid for the five models are listed in Table 2. As with the models of Espinasse \& Kimber (1981) the relative affinity of the more-related genomes is defined as $x$, and the less-related genomes as $y$, where $x>y$ and $x+y=1$. In four cases (the $2: 2: 1,3: 1: 1,2: 1: 1: 1$ and $4: 1$ models) the relative affinity is expressed directly in the pairing ratio between the more versus the less closely related genomes (Table 2). For the 3:2 model, however, this interpretation is inadequate as genomes 1,2 and 3 each have a 'choice' of two more related pairing partners, whereas genomes 4 and 5 have a 'choice' of only one. In consequence the probability of pairing between, for example, genomes 1 and 2 will always be less than between 3 and 4 , although their relative affinities are the same. To account for this in

Table 2 Relative affinities, and proportions of total pairing $\left(f_{i}\right)$ among the genomes of a pentaploid

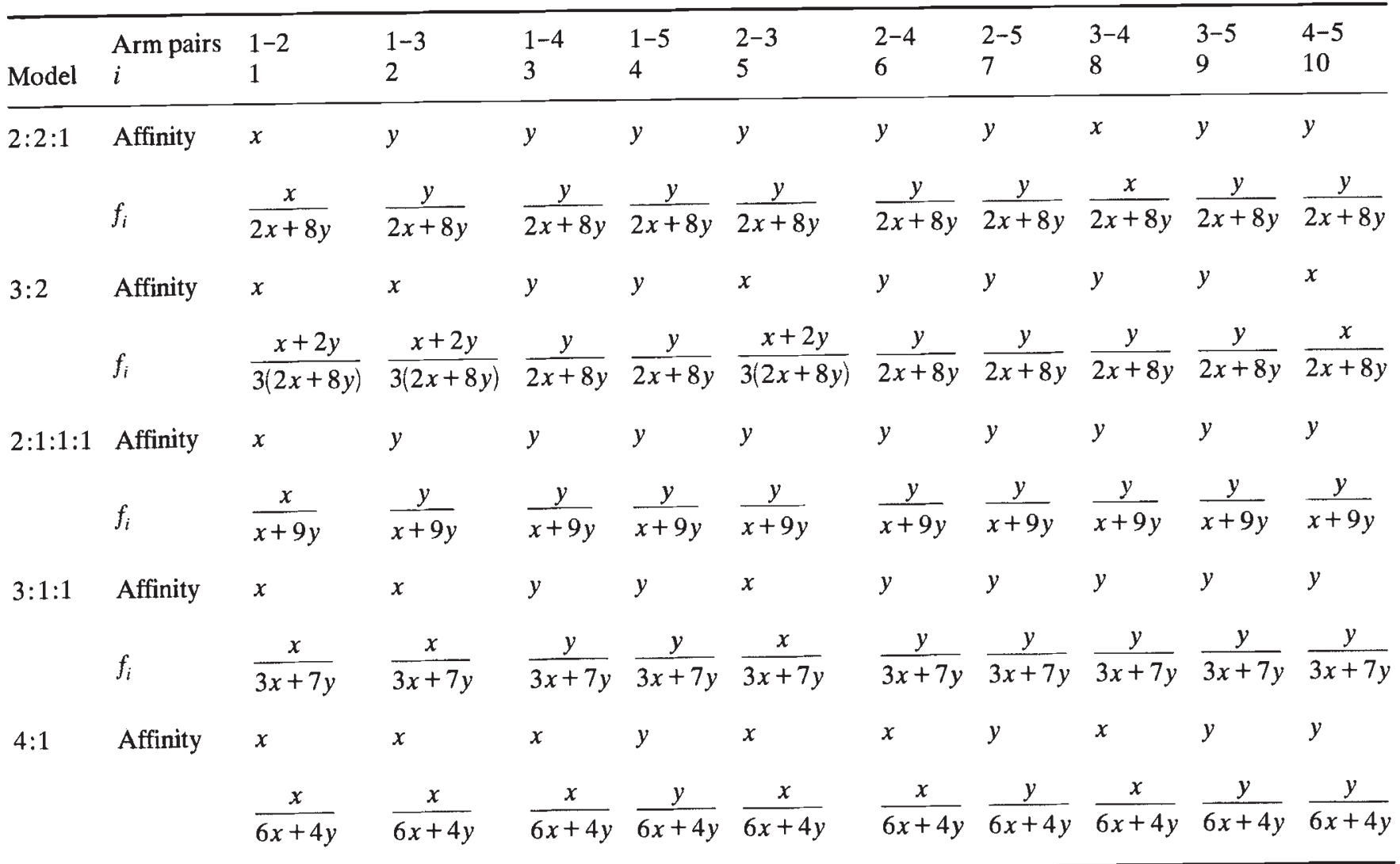


the calculations the amount of pairing within each of the three possible pairs in the triplet of genomes is expressed as $(x+2 y) / 3$ (Table 2). This returns a value of 0.33 when $x=1$, and 0.50 when $x=0.5$ and pairing is random among the genomes (Table 2).

With partner exchange excluded by definition, there are 15 possible pairing patterns for five homoeologous chromosome arms made up of two pairs of arms plus one unpaired arm, e.g. $(1 \sim 2,3 \sim 4,5)$ where arms 1 and 2 , and 3 and 4 are paired, arm 5 is unpaired, and the symbol ' $\sim$ ' indicates possible rather than realized pairing as seen at metaphase I. Viewing the pairing of arms as a sequential process, there are two ways in which this pattern might be achieved, either through the formation of the $1 \sim 2$ pair followed by the $3 \sim 4$ pair, or in reverse order. The probability of the first pair being $1 \sim 2$ is $f_{1}$ (see Table 2), and the conditional probability of the second being $3 \sim 4$ from among the three remaining possible pairs is $f_{8} / g_{8}$, where $g_{8}=f_{8}+f_{9}+f_{10}$ and $f_{8}, f_{9}$ and $f_{10}$ are the proportions of total pairing due to the $3 \sim 4,4 \sim 5$ and $3 \sim 5$ pairs respectively. Similar reasoning applies to the formation of the pattern in the reverse order. Thus the total proportion of the $(1 \sim 2,3 \sim 4,5)$ pattern is $v_{1}=f_{1} f_{8} /$ $g_{8}+f_{8} f_{1} / g_{1}=f_{1} f_{8}\left(1 / g_{1}+1 / g_{8}\right)$. By the same process the proportion of the other 14 patterns, $v_{2 \ldots 15}$, may be determined. Each arm pair can occur in three pairing patterns. Thus the probability of arm pair $(1 \sim 2)$ in all patterns is $h_{1}=v_{1}+v_{2}+v_{3}$, and similarly for the other nine possible arm pairs.

For a pentaploid hybrid, $c$, the mean arm-pairing frequency may be determined from an observed dataset as

$c=[\lfloor$ II $\rfloor+2($ II $)+$ III $)+3\lfloor$ IV $\rfloor+4($ IV $)+\mathrm{V}] / 4 b$

where: $\lfloor$ II $\rfloor=$ rod bivalents, (II) $=$ ring bivalents, III = trivalents, $\lfloor$ IV $\rfloor=$ open quadrivalents, IV = closed quadrivalents, $\mathbf{V}=$ quinquevalents, $b=$ basic number.

With relative affinity reflected in the ratio of pairing among arms and the number of pairings expected in any set of five arms as $2 c$, each arm pair is really formed with a frequency of $2 c f_{i}$. Hence the probability of any notional arm pair really being paired as seen at metaphase I is $p_{i}=2 c f_{i} / h_{i}$, and that of it being unpaired $q_{i}=1-p_{i}$.

From this situation it is possible to calculate the contribution of each pairing pattern to the frequency of the 26 arm configurations $\left(A_{1 \ldots 26}\right)$, namely, 15 combinations of two sets of paired arms, e.g. $(1-3,4-5,2) ; 10$ combinations of one set of paired arms, e.g. $(2-3,1,4$, $5)$; and 1 combination of no pairs of arms, (1, 2, 3, 4, $5)$; with '-' indicating realized pairing observed at metaphase I of meiosis. For example, within the $(1 \sim 2,3 \sim$ $4,5)$ pattern the contribution to the frequency of arm configuration $(1-2,3-4,5)=v_{1} p_{1} p_{8} ;$ to $(1-2,3,4$, $5)=v_{1} p_{1} q_{8}$; to $(3-4,1,2,3)=v_{1} q_{1} p_{8}$; and to $(1,2,3$, $4,5)=v_{1} q_{1} q_{8}$.

The 12 meiotic configurations that a single homoeologous group of five chromosomes can form ranges from five univalents to a quinquevalent, a ring quadrivalent and a univalent, or two ring bivalents and a univalent, depending upon the pattern and amount of pairing. Their frequencies may be determined by establishing a $26 \times 26$ array and siting the arm configurations and their frequencies along the margins. For each of the 676 cells within the array the resulting configuration can be established from the marginal arm configurations, and its frequency as the product of the marginal frequencies. Summing for each configuration type over the whole array gives its frequency, and hence the frequencies of each meiotic figure (univalents through quinquevalents).

The optimization process to determine the best estimate of $x$ is performed as described for the triploid model (Chapman \& Kimber, 1992a) by first calculating $c$ from the observed frequencies of figures, and then searching the permissible range of $x(0.5-1.0)$ for that which gives the minimum weighted sums of squares of difference (WSSD) between the observed and calculated number of each figure type. The weighting factor used is the number of chromosomes in each figure.

\section{Results and discussion}

When applied to the datasets constructed for ideal $5: 0$, $2: 2: 1,3: 2,2: 1: 1: 1,3: 1: 1$ and $4: 1$ hybrids with $x=1$, the respective models give exact fits with WSSDs of zero and $x$ also equal to one (see Table 1 ), and there is no second solution for the ideal 3:2 dataset. Additionally, and unlike the equations of Espinasse \& Kimber (1981), when $x=1, c$ cannot exceed 0.5 for the $2: 1: 1: 1$ and $3: 1: 1$ models, for then negative, and hence irrational, numbers of meiotic figures are calculated. This is in agreement with the theoretical consideration that pairing should be confined entirely to the more closely related genomes.

Figure 2 shows how the numbers of figures vary with $x$ when $c=0.929$ for the $2: 2: 1$ and $3: 2$ models and $c=0.5$ for the $2: 1: 1: 1$ and $3: 1: 1$ models, when the basic number $=7$ for all four models. These values have been chosen for direct comparison with plots in Figs 1 and 2 of Espinasse \& Kimber (1981). The present figures show a broad similarity to those of Espinasse \& Kimber (1981) but differ in detail. For example here the trivalents in the 2:1:1 model decline smoothly from $x=0.5$ to 1.0 rather than showing a peak around $x=0.9$. In the $3: 1: 1$ model the calculated number of trivalents exceeds those of rod bivalents 

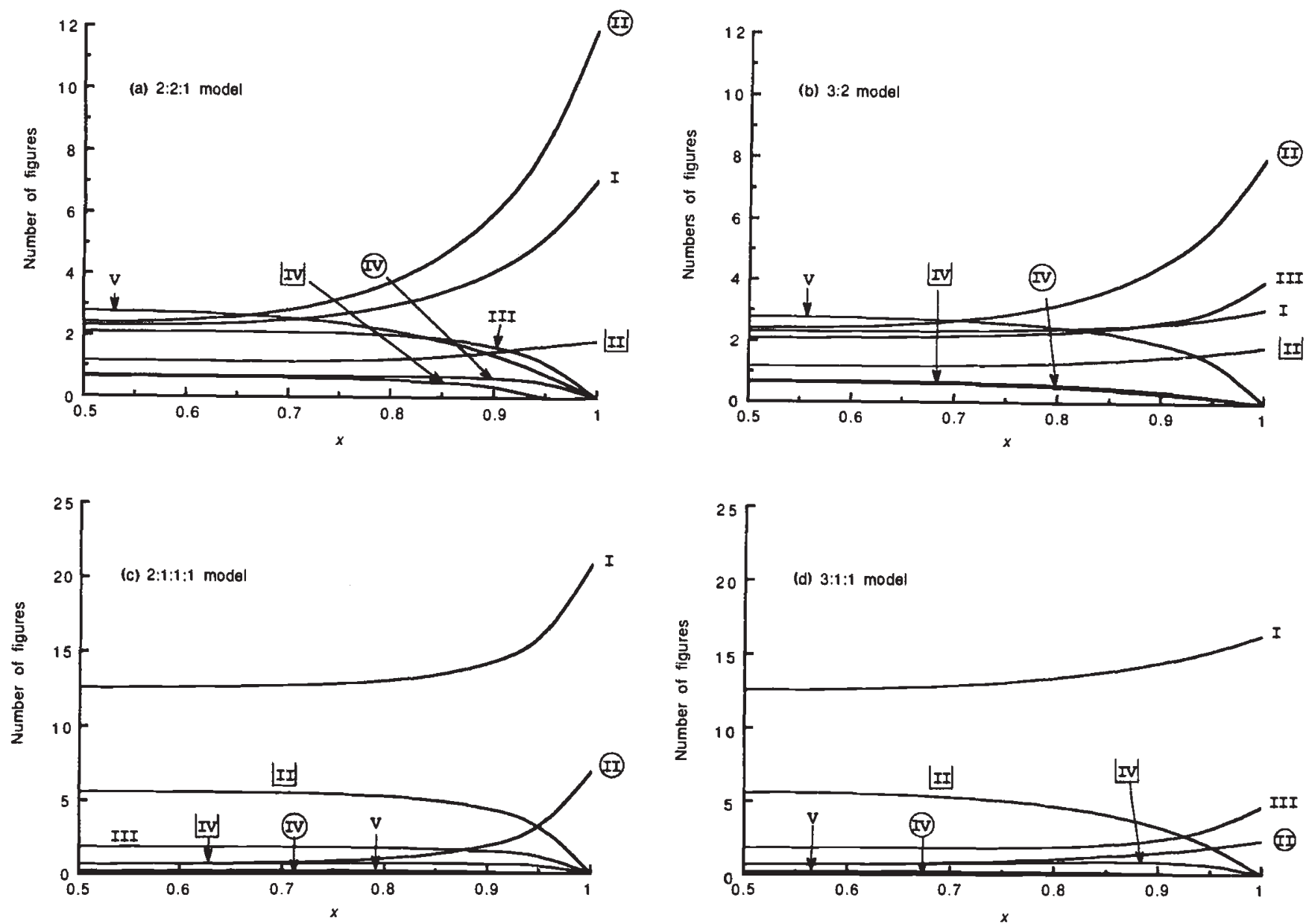

Fig. 2 The variation of expected numbers of meiotic figures with $x$ for the 2:2:1 (a) and 3:2 (b) models, when $c=0.929$; and for the 2:1:1:1 (c) and 3:1:1 (d) models when $c=0.500$.

from values of $x$ around 0.92 upwards rather than remaining less than rod bivalents throughout the full permissible range of $x$. Such differences may lead to differing interpretations for some real datasets.

As with the triploid and tetraploid models, the expected figure numbers change slowly at the lower values of $x$, especially if $c$ is also low, and there is little difference among these numbers for the various models. In practice this means that at low values of $c$ it may not be possible to make a decisive choice of a best-fit model unless the value of $x$ is high, e.g. in excess of 0.9 . When the best fit solutions to several models give similar WSSDs and low values of $x$, the best interpretation is that all five genomes are similarly differentiated from each other.

Table 3 compares analyses using the present equations with a representative sample of those carried out by Espinasse \& Kimber (1981). As they found, there were no cases of the $4: 1$ model as a best solution and this has therefore been omitted from the table. It should be noted that the merit functions calculated for the different approaches are not comparable, due to the weighting applied in the present approach.

In the first two examples there is strong agreement that the $2: 2: 1$ model is the best solution. In the first case it is known from the pedigree of the hybrid that there should be two copies of the two genomes A and $\mathrm{G}$ from Triticum timopheevii, whilst the fifth is from Hordeum bogdanii, a taxonomically remote species whose $\mathrm{H}$ genome is unlikely to demonstrate homology in pairing with those of Triticum. A somewhat similar argument applies to the $T$. aestivum $\times T$. turgidum hybrid, except that the hexaploid parent is a natural allopolyploid thought to be of comparatively recent origin from the hybridization of $T$. turgidum $\times T$. tauschii. The best fit 2:2:1 model, with high values of $c$ and $x$ are in full agreement with this view.

The two $T$. aestivum $\times T$. timopheevii hybrids show a greater consistency in the best solution proposed by the present analysis compared to that of Espinasse \& 
Table 3 Comparison of the present analyses of pentaploid hybrids with those of Espinasse \& Kimber (1980)

\begin{tabular}{|c|c|c|c|c|c|c|c|}
\hline \multirow[b]{2}{*}{ Hybrid and genomes } & \multirow[b]{2}{*}{$c$} & & & \multicolumn{4}{|l|}{ Models } \\
\hline & & & & $2: 2: 1$ & $3: 2$ & $2: 1: 1: 1$ & $3: 1: 1$ \\
\hline \multirow{4}{*}{$\begin{array}{l}\text { 1. (Triticum timopheevii } \times \\
\text { Hordeum bogdanii) } \times \\
\text { T. timopheevii AAGGH }\end{array}$} & \multirow[t]{4}{*}{0.970} & $\mathrm{E} / \mathrm{K}$ & SSD & 0.002 & 58.33 & 47.97 & 125.2 \\
\hline & & & $x$ & 0.998 & 1.000 & 1.000 & 1.000 \\
\hline & & $\mathrm{C} / \mathrm{K}$ & WSSD & 0.059 & 254.74 & 426.74 & 610.29 \\
\hline & & & $x$ & 0.999 & 0.996 & $0.897^{*}$ & $0.788^{*}$ \\
\hline \multirow{4}{*}{$\begin{array}{l}\text { 2. T. aestivum } \times \\
\text { T. turgidum AABBD }\end{array}$} & \multirow[t]{4}{*}{0.885} & $\mathrm{E} / \mathrm{K}$ & SSD & 0.006 & 48.06 & 19.84 & 83.53 \\
\hline & & & $x$ & 0.999 & 1.000 & 1.000 & 1.000 \\
\hline & & $\mathrm{C} / \mathrm{K}$ & WSSD & 0.042 & 180.15 & 328.17 & 451.43 \\
\hline & & & $x$ & 0.999 & 0.999 & 0.894 & $0.764^{*}$ \\
\hline \multirow{4}{*}{$\begin{array}{l}\text { 3. T. aestivum } \times \\
\text { T. timopheevii } \mathrm{AABGD}\end{array}$} & \multirow[t]{4}{*}{0.578} & $\mathrm{E} / \mathrm{K}$ & SSD & 0.060 & 3.144 & 4.063 & 11.03 \\
\hline & & & $x$ & 0.947 & 1.000 & 0.940 & 0.771 \\
\hline & & $\mathrm{C} / \mathrm{K}$ & WSSD & 2.622 & 4.362 & 43.751 & 55.285 \\
\hline & & & $x$ & 0.954 & 1.000 & 0.868 & 0.631 \\
\hline \multirow{4}{*}{$\begin{array}{l}\text { 4. T. aestivum } \times \\
\text { T. timopheevii AABGD }\end{array}$} & \multirow[t]{4}{*}{0.570} & $E / K$ & SSD & 1.642 & 6.312 & 1.177 & 9.796 \\
\hline & & & $x$ & 0.950 & 1.000 & 0.958 & 0.899 \\
\hline & & $\mathrm{C} / \mathrm{K}$ & WSSD & 9.489 & 14.242 & 29.594 & 52.127 \\
\hline & & & $x$ & 0.950 & 0.993 & 0.901 & 0.745 \\
\hline \multirow{4}{*}{$\begin{array}{l}\text { 5. T. aestivum } \times \\
\text { (T. boeoticum } \times \\
\text { T. urartu) AAABD }\end{array}$} & \multirow[t]{4}{*}{0.335} & $\mathrm{E} / \mathrm{K}$ & SSD & 8.944 & 8.948 & 2.934 & 0.376 \\
\hline & & & $x$ & 0.543 & 0.500 & 0.972 & 1.000 \\
\hline & & $\mathrm{C} / \mathrm{K}$ & WSSD & 35.354 & 35.354 & 17.544 & 1.586 \\
\hline & & & $x$ & 0.500 & 0.500 & 0.957 & 0.992 \\
\hline \multirow{4}{*}{$\begin{array}{l}\text { 6. T. crassum } \times \\
\text { T. cylindricum } \mathrm{CDDDM}\end{array}$} & \multirow[t]{4}{*}{0.562} & $\mathrm{E} / \mathrm{K}$ & SSD & 5.862 & 11.20 & 0.030 & 7.244 \\
\hline & & & $x$ & 0.942 & 0.943 & 0.967 & 0.962 \\
\hline & & $\mathrm{C} / \mathrm{K}$ & WSSD & 21.222 & 30.730 & 11.861 & 39.607 \\
\hline & & & $x$ & 0.934 & 0.968 & 0.919 & 0.820 \\
\hline \multirow{4}{*}{$\begin{array}{l}\text { 7. T. crassum } \times T \text {. kotschyi } \\
\text { DDMS } U\end{array}$} & \multirow[t]{4}{*}{0.353} & $E / K$ & SSD & 3.888 & 4.276 & 0.109 & 1.110 \\
\hline & & & $x$ & 0.868 & 0.763 & 0.958 & 0.945 \\
\hline & & $\mathrm{C} / \mathrm{K}$ & WSSD & 12.988 & 14.399 & 0.482 & 5.266 \\
\hline & & & $x$ & 0.856 & 0.808 & 0.944 & 0.901 \\
\hline \multirow{4}{*}{$\begin{array}{l}\text { 8. T. juvenale } \times \\
\text { T. cylindricum CDDMU }\end{array}$} & \multirow[t]{4}{*}{0.458} & $\mathrm{E} / \mathrm{K}$ & SSD & 0.080 & 0.080 & 0.660 & 0.825 \\
\hline & & & $x$ & 0.838 & 0.885 & 0.811 & 0.500 \\
\hline & & $\mathrm{C} / \mathrm{K}$ & WSSD & 0.325 & 0.181 & 2.014 & 2.396 \\
\hline & & & $x$ & 0.826 & 0.874 & 0.771 & 0.500 \\
\hline
\end{tabular}

For data sources see Espinasse \& Kimber (1981). *Calculated figure numbers include some negative frequencies.

Kimber (1981). Both agree on the 2:2:1 model for example 3 , but for example 4 the earlier analysis favours a $2: 1: 1: 1$ model, whereas the present one clearly excludes it, favouring the $2: 2: 1$ model instead.

With the $T$. aestivum $\times(T$. boeoticum $\times T$. urartu $)$ hybrid there is clear agreement on the $3: 1: 1$ model. This does not necessarily mean that the three $A$ genomes present from the different species should be considered identical. Rather, they are comparatively close and similarly differentiated when compared with the $\mathrm{B}$ and $\mathrm{D}$ genomes.

In the sixth example, hexaploid $T$. crassum $\times T$. cylindricum, there is agreement on the $2: 1: 1: 1$ model giving the best fit, but whilst that of Espinasse \& Kimber appears good, the present fit is poor with a lower value of $x$. There is no indication that the hybrid might fit the $3: 1: 1$ model, as the genomic formula would suggest, and this accords with Kimber \& Zhao's (1983) findings that the D genomes in T. crassum are differentiated from those in T. cylindricum and would pair preferentially with each other. This is also consistent with the 2:1:1:1 model being clearly the best fit for the hexaploid T. crassum $\times$ T. kotschyi hybrid, the seventh example.

The final example should also fit a 2:1:1:1 model, but does not in either analysis, giving reasonable fits to both the $2: 2: 1$ and $3: 2$ models. However, with a $c$ value of less than 0.5 and an $x$ value less than 0.9 , this puts the analysis in a region where there is little to choose between the models. The best explanation for the behaviour of the $T$. juvenale $\times T$. cylindricum hybrid is that all five genomes are more or less equally 
differentiated. This again is in agreement with the conclusion of Kimber \& Zhao (1983) that the D genome in T. juvenale is substantially modified.

Among the hybrids considered here there is broad agreement between the present analyses and those of Espinasse \& Kimber (1981), but there are some differences in detail. More important perhaps are the inherent problems in dealing with meiotic analyses at the pentaploid level. The greater number of available models increases the likelihood that any observed dataset will find a tolerably good fit. Furthermore, at low levels of pairing the models tend to predict rather similar frequencies of meiotic figures unless the values of $x$ are high. When the dependability of the assumptions on which the models are based is also considered, then only cautious conclusions should be drawn from these pentaploid models unless the best fits are clearcut or there is additional supporting evidence.

\section{A note on programs}

All the programs for the analysis of meiosis in species hybrids described in this series of papers have been written in TURBO PASCAL to run on the Apple Macintosh. Copies of the source code and the compiled programs are available from the authors upon receipt of an $800 \mathrm{~K}$ or $1.4 \mathrm{MB}$ initialized disk. Use of the source code requires TURBO PASCAL.

\section{Acknowledgements}

CGDC was supported by a post-doctoral fellowship from the University of Missouri. Contribution from the Missouri Agricultural Experiment Station. Journal Series Number: 11,259.

\section{References}

ALONSO, L. C. AND KIMBER, G. 1981. The analysis of meiosis in hybrids. II. Triploid hybrids. Can. J. Genet. Cytol., 23, 221-234.

CHAPMAN, C. G. D. AND KIMBER, G. 1992a. Developments in the meiotic analysis of hybrids. I. Review of theory and optimization in triploids. Heredity, 68 97-103.

CHAPMAN, C. G. D. AND KIMBER, G. 1992b. Developments in the meiotic analysis of hybrids. II. Amended models for tetraploids. Heredity, 68 105-113.

ESPINASSE, A. AND KIMBER, G. 1981. The analysis of meiosis in hybrids. IV. Pentaploid hybrids. Can. J. Genet. Cytol., 23, 627-638 (see also erratum in Can. J. Genet. Cytol., 26, 229).

KIMBER, G. AND ALONSO, L. C. 1981. The analysis of meiosis in hybrids. III. Tetraploid hybrids. Can. J. Genet. Cytol., 23, 235-254.

KIMBER, G. AND YEN, Y. 1990. The genomic analysis of diploid plants. Proc. Natl. Acad. Sci., USA, 87, 3205-3209.

KIMBER, G. AND ZHAO, Y. H. 1983. The D genome of the Triticeae. Can. J. Genet. Cytol., 25, 581-589.

\section{Appendix}

Tables A1-3 below lay out in detail the calculations for the pentaploid models from the relative affinities through to the frequencies of the arm configurations. The step numbers above some columns indicate the sequence of calculation.

Table A1 Calculations relating to specific arm pairs

\begin{tabular}{|c|c|c|c|c|c|c|}
\hline \multirow[b]{3}{*}{$i$} & \multirow[b]{3}{*}{ Arm pair } & \multicolumn{5}{|l|}{ Step } \\
\hline & & 1 & 2 & 4 & 5 & 6 \\
\hline & & $\begin{array}{c}\text { Proportion of } \\
\text { total pairing } \\
\text { (see Table 1) } \\
f_{i}\end{array}$ & $\begin{array}{c}\Sigma \text { probabilities of } \\
\text { other three arms } \\
g_{i}\end{array}$ & $\begin{array}{l}\text { Probability of } \\
\text { possible arm pairs } \\
\left(v_{j} \text { from Table A2) }\right. \\
h_{i}\end{array}$ & $\begin{array}{l}\text { Probability of } \\
\text { possible arm pair } \\
\text { being paired at } \\
\text { metaphase I } \\
\qquad p_{i}\end{array}$ & $\begin{array}{l}\text { Probability of } \\
\text { possible arm pair } \\
\text { being unpaired at } \\
\text { metaphase I } \\
\qquad q_{i}\end{array}$ \\
\hline 1 & $1 \sim 2$ & $f_{1}$ & $f_{8}+f_{9}+f_{10}$ & $v_{1}+v_{2}+v_{3}$ & $2 c f_{1} / h_{1}$ & $1-p_{1}$ \\
\hline 2 & $1 \sim 3$ & $f_{2}$ & $f_{6}+f_{7}+f_{10}$ & $v_{4}+v_{5}+v_{6}$ & $2 c f_{2} / h_{2}$ & $1-p_{2}$ \\
\hline 3 & $1 \sim 4$ & $f_{3}$ & $f_{5}+f_{7}+f_{9}$ & $v_{7}+v_{8}+v_{9}$ & $2 c f_{3} / h_{3}$ & $1-p_{3}$ \\
\hline 4 & $1 \sim 5$ & $f_{4}$ & $f_{5}+f_{6}+f_{8}$ & $v_{10}+v_{11}+v_{12}$ & $2 c f_{4} / h_{4}$ & $1-p_{4}$ \\
\hline 5 & $2 \sim 3$ & $f_{5}$ & $f_{3}+f_{4}+f_{10}$ & $v_{13}+v_{14}+v_{15}$ & $2 c f_{5} / h_{5}$ & $1-p_{5}$ \\
\hline 6 & $2 \sim 4$ & $f_{6}$ & $f_{2}+f_{4}+f_{9}$ & $v_{4}+v_{11}+v_{14}$ & $2 c f_{6} / h_{6}$ & $1-p_{6}$ \\
\hline 7 & $2 \sim 5$ & $f_{7}$ & $f_{2}+f_{3}+f_{8}$ & $v_{5}+v_{8}+v_{15}$ & $2 c f_{7} / h_{7}$ & $1-p_{7}$ \\
\hline 8 & $3 \sim 4$ & $f_{8}$ & $f_{1}+f_{4}+f_{7}$ & $v_{1}+v_{12}+v_{15}$ & $2 c f_{8} / h_{8}$ & $1-p_{8}$ \\
\hline 9 & $3 \sim 5$ & $f_{9}$ & $f_{1}+f_{3}+f_{6}$ & $v_{2}+v_{9}+v_{14}$ & $2 c f_{9} / h_{9}$ & $1-p_{9}$ \\
\hline 10 & $4 \sim 5$ & $f_{10}$ & $f_{1}+f_{2}+f_{5}$ & $v_{3}+v_{6}+v_{13}$ & $2 c f_{10} / h_{10}$ & $1-p_{10}$ \\
\hline
\end{tabular}


Table A2 Calculation of pairing pattern frequencies

\begin{tabular}{rll}
\hline & & Step 3 \\
\cline { 3 - 3 } & & $\begin{array}{l}\text { Probability of pairing } \\
\text { pattern }\left(f_{i} \text { and } g_{i} \text { from }\right.\end{array}$ \\
& Possible & Table A1 $)$ \\
& pairing pattern & \multicolumn{1}{c}{$v_{j}$} \\
\hline & $1 \sim 2,3 \sim 4,5$ & $f_{1} f_{8}\left(1 / g_{1}+1 / g_{8}\right)$ \\
\hline 1 & $1 \sim 2,3 \sim 5,4$ & $f_{1} f_{9}\left(1 / g_{1}+1 / g_{9}\right)$ \\
3 & $1 \sim 2,4 \sim 5,3$ & $f_{1} f_{10}\left(1 / g_{1}+1 / g_{10}\right)$ \\
4 & $1 \sim 3,2 \sim 4,5$ & $f_{2} f_{6}\left(1 / g_{2}+1 / g_{6}\right)$ \\
5 & $1 \sim 3,2 \sim 5,4$ & $f_{2} f_{7}\left(1 / g_{2}+1 / g_{7}\right)$ \\
6 & $1 \sim 3,4 \sim 5,2$ & $f_{2} f_{10}\left(1 / g_{2}+1 / g_{10}\right)$ \\
7 & $1 \sim 4,2 \sim 3,5$ & $f_{3} f_{5}\left(1 / g_{3}+1 / g_{5}\right)$ \\
8 & $1 \sim 4,2 \sim 5,3$ & $f_{3} f_{7}\left(1 / g_{3}+1 / g_{7}\right)$ \\
9 & $1 \sim 4,3 \sim 5,2$ & $f_{3} f_{9}\left(1 / g_{3}+1 / g_{9}\right)$ \\
10 & $1 \sim 5,2 \sim 3,4$ & $f_{4} f_{5}\left(1 / g_{4}+1 / g_{5}\right)$ \\
11 & $1 \sim 5,2 \sim 4,3$ & $f_{4} f_{6}\left(1 / g_{4}+1 / g_{6}\right)$ \\
12 & $1 \sim 5,3 \sim 4,2$ & $f_{4} f_{8}\left(1 / g_{4}+1 / g_{8}\right)$ \\
13 & $2 \sim 3,4 \sim 5,1$ & $f_{5} f_{10}\left(1 / g_{5}+1 / g_{10}\right)$ \\
14 & $2 \sim 4,3 \sim 5,1$ & $f_{6} f_{9}\left(1 / g_{6}+1 / g_{9}\right)$ \\
15 & $2 \sim 5,3 \sim 4,1$ & $f_{7} f_{8}\left(1 / g_{7}+1 / g_{8}\right)$ \\
\hline
\end{tabular}

Table A3 Calculation of frequency of arm configurations $\left(A_{k}\right)$

\begin{tabular}{|c|c|c|c|c|c|}
\hline \multirow[b]{2}{*}{$k$} & \multirow[b]{2}{*}{$\begin{array}{l}\text { Arm } \\
\text { configuration }\end{array}$} & \multicolumn{2}{|l|}{ Step 7} & \multirow[b]{2}{*}{$\begin{array}{l}\text { Arm } \\
\text { configuration }\end{array}$} & \multirow{2}{*}{$\begin{array}{l}\text { Step } 7 \\
\text { Frequency } \\
A_{k}\end{array}$} \\
\hline & & $\begin{array}{l}\text { Frequency } \\
A_{k}\end{array}$ & $k$ & & \\
\hline 1 & $1-2,3-4,5$ & $v_{1} p_{1} p_{8}$ & 16 & $1-2,3,4,5$ & $p_{1}\left(v_{1} q_{8}+v_{2} q_{9}+v_{3} q_{10}\right)$ \\
\hline 2 & $1-2,3-5,4$ & $v_{2} p_{1} p_{9}$ & 17 & $1-3,2,4,5$ & $p_{2}\left(v_{4} q_{6}+v_{5} q_{7}+v_{6} q_{10}\right)$ \\
\hline 3 & $1-2,4-5,3$ & $v_{3} p_{1} p_{10}$ & 18 & $1-4,2,3,5$ & $p_{3}\left(v_{7} q_{5}+v_{8} q_{7}+v_{9} q_{9}\right)$ \\
\hline 4 & $1-3,2-4,5$ & $v_{4} p_{2} p_{6}$ & 19 & $1-5,2,3,4$ & $p_{4}\left(v_{10} q_{5}+v_{11} q_{6}+v_{12} q_{8}\right)$ \\
\hline 5 & $1-3,2-5,4$ & $v_{5} p_{2} p_{7}$ & 20 & $2-3,1,4,5$ & $p_{5}\left(v_{7} q_{3}+v_{10} q_{4}+v_{13} q_{10}\right)$ \\
\hline 6 & $1-3,4-5,2$ & $v_{6} p_{2} p_{10}$ & 21 & $2-4,1,3,5$ & $p_{6}\left(v_{4} q_{2}+v_{11} q_{4}+v_{14} q_{9}\right)$ \\
\hline 7 & $1-4,2-3,5$ & $v_{7} p_{3} p_{5}$ & 22 & $2-5,1,3,4$ & $p_{7}\left(v_{5} q_{2}+v_{8} q_{3}+v_{15} q_{8}\right)$ \\
\hline 8 & $1-4,2-5,3$ & $v_{8} p_{3} p_{7}$ & 23 & $3-4,1,2,5$ & $p_{8}\left(v_{1} q_{1}+v_{12} q_{4}+v_{15} q_{7}\right)$ \\
\hline 9 & $1-4,3-5,2$ & $v_{9} p_{3} p_{9}$ & 24 & $3-5,1,2,4$ & $p_{9}\left(v_{2} q_{1}+v_{9} q_{3}+v_{14} q_{6}\right)$ \\
\hline 10 & $1-5,2-3,4$ & $v_{10} p_{4} p_{5}$ & 25 & $4-5,1,2,3$ & $p_{10}\left(v_{3} q_{1}+v_{6} q_{2}+v_{13} q_{5}\right)$ \\
\hline 11 & $1-5,2-4,3$ & $v_{11} p_{4} p_{6}$ & & & \\
\hline 12 & $1-5,3-4,2$ & $v_{12} p_{4} p_{8}$ & 26 & $1,2,3,4,5$ & $1-\Sigma A_{1 \ldots 25}$ \\
\hline $\begin{array}{l}13 \\
14\end{array}$ & $\begin{array}{l}2-3,4-5,1 \\
2-4,3-5,1\end{array}$ & $\begin{array}{l}v_{13} p_{5} p_{10} \\
v_{14} p_{6} p_{9}\end{array}$ & & & \\
\hline 15 & $2-5,3-4,1$ & $v_{15} p_{7} p_{8}$ & & & \\
\hline
\end{tabular}

